\title{
Influenza in the Eastern Mediterranean Region: identifying the unknowns for detection and control of epidemic and pandemic threats
}

\author{
Mamunur Malik', Jaouad Mahjour ${ }^{2}$, Wasiq Khan ${ }^{1}$ and Ala Alwan ${ }^{3}$
}

Acute respiratory illnesses and influenza-like illnesses (ILI) are a significant cause of morbidity and mortality worldwide. Data from developed countries reveal that seasonal influenza can affect up to $15 \%$ of the population presenting with upper respiratory tract infections and may result in up to 500000 deaths worldwide annually (1). Despite their public health importance, little was known about the aetiology of these illnesses in the countries of the WHO Eastern Mediterranean Region (EMR).

Since the emergence of highly pathogenic avian influenza A (H5N1) virus in Egypt in 2006, the WHO Regional Office for the Eastern Mediterranean has been working closely with the countries of the Region to establish and enhance sentinel surveillance systems for ILI and severe acute respiratory infections (SARI). The United States Centers for Disease Control and Prevention (US-CDC) and the Global Disease Detection and Response Programme of the US Naval Medical Research Unit no 3 (NAMRU-3) based in Cairo, Egypt have been collaborating with $\mathrm{WHO}$ to strengthen national capacity in two broad areas - laboratory diagnostics capacity to detect seasonal and novel influenza viruses and epidemiological surveillance for ILI and SARI to improve understanding of the epidemiology, seasonality and risk factors for influenza-associated illnesses. This collaborative programme has helped establish functional sentinel surveillance systems for ILI and SARI in 15 of the 22 countries in the Region, including having 16 designated national influenza centres in 15 countries.

The Pandemic Influenza Preparedness (PIP) Framework has provided further incentives to the countries in the Region to enhance surveillance for seasonal influenza and optimize the use of surveillance data for public-health decision making. As a result, countries have now conducted disease burden studies using data from the populationbased surveillance system for influenza and acute respiratory infections.

What we are seeing now is that these investments are paying rich dividends. A wealth of new information and evidence has started to emerge from EMR countries, such as the types and patterns of seasonal influenza viruses circulating in the Region, seasonality of these circulating viruses, and also the burden of influenza-associated illnesses and the risk factors which had remained largely unknown.

This theme issue focusing on influenza research in the EMR includes research articles on disease burden associated with influenza from Egypt, Islamic Republic of Iran and Tunisia. They present population-based estimates of the burden of influenza-associated illnesses using a standardized and consistent approach characterizing the burden by age, demographic and clinical characteristics as well as by discernible seasonal variables. The methods used allow the findings on the influenza disease incidence to be compared across other countries in the Region with the same climatic and demographic characteristics.

The issue also includes a number of articles that demonstrate the improved knowledge on the types and patterns of seasonal influenza viruses circulating in the Region. Data from Afghanistan, Egypt, Islamic Republic of Iran, Lebanon, Morocco and Yemen, collected over a substantial period of time, show that influenza $A$ and $B$ viruses predominantly circulate throughout the year with both the influenza A (H1N1) pdm09 and Influenza A (H3N2) viruses being associated with severity and higher rates of complications, including increased hospitalizations and deaths. Data from Egypt and Morocco show that respiratory syncytial virus is also an important etiological agent for acute lower respiratory tract infections among those aged under five years, resulting in considerable hospitalization.

While these pioneering works are contributing to our knowledge and understanding of the epidemiology of influenza in the Region, including its seasonality and risk factors, our next priority is to use the information to develop appropriate policies for influenza preventive and control. Involvement of relevant decisions-makers and policy 
planners in the countries is essential in order to translate the evidence into concrete policy actions for introduction and increased use of seasonal influenza vaccines or other pharmacological control interventions, such as the early use of antivirals. Substantial evidence exists on the effectiveness of seasonal influenza vaccines in preventing hospitalizations especially in high risk age groups (2), and the data generated on the burden of influenza-associated illnesses among different age groups will be extremely valuable in selecting and targeting specific age groups for seasonal influenza vaccines.

One of the biggest challenges in the Region continues to be early detection of influenza epidemics because the timing and severity of epidemics and the distribution of circulating viruses are variable and difficult to predict (3). Addressing this requires countries to conduct time-series analyses of their own SARI surveillance data and set their own national baseline threshold values in order to predict and detect aberrations which may trigger the onset of an influenza epidemic. Egypt has estimated its thresholds for seasonal influenza using a simple technique recommended by WHO (4). This provides a good example for other countries to replicate in order to set their own national baseline thresholds.

Even though our knowledge on influenza unknowns has improved in the Region to allow better and informed decision-making, new threats emerge. Several newly discovered respiratory viruses, such avian influenza virus A (H5N1), avian influenza A(H9N2) , swine-origin influenza A (H1N1), and the Middle East respiratory syndromecoronavirus (MERS-CoV), which are mostlyzoonotic in origin, have emerged in the Region since 2006 posing severe epidemic and pandemic threats (5). The emergence and continued transmission of these respiratory viruses in the Region emphasize the importance of having strengthened surveillance systems for SARI and ILI in every country in order to early detect and promptly respond to the emergence of any novel influenza virus in human populations that may threaten the regional health security. The emergence of such viruses is expected but highly unpredictable in terms of time of occurrence and ability to cause severe infection. This was seen during the pandemic swineorigin influenza A (H1N1) of 2009 which was marked by two outstanding characteristics: the ability of the virus to cause major out-of-season epidemics in temperate countries and to cause unusually severe disease and death among the young and healthy people (6). Data available from countries outside our Region also indicate that the public health benefits of strong surveillance systems for SARI and ILI can go beyond influenza to other emerging diseases. Such influenza surveillance platforms can be used to respond to other emerging health threats such as MERS (7).

Threats from zoonotic influenza remain and seasonal influenza epidemics and pandemic influenza are expected though unpredictable events. It is imperative therefore that sentinel surveillance systems for influenza are sustained within a country's routine surveillance programme (8). These systems provide the needed infrastructure to detect and respond to the threats at the national level and help improve global detection and response and safeguard global public health security.

\section{References}

1. World Health Organization. Influenza (seasonal) Fact Sheet. March 2014 (http://www.who.int/mediacentre/factsheets/ fs211/en, accessed 23 June 2016).

2. Vaccines against influenza. WHO position paper - November 2012. Wkly Epidemiol Rec, 2012. 87(47):461-76.

3. Bresee J, Hayden FG. Epidemic influenza - responding to the expected but unpredictable. N Engl J Med, 2013. 368(7):58992.

4. Global Epidemiological Surveillance Standards for Influenza. World Health Organization 2013 (http://www.who.int/influenza/resources/documents/WHO_Epidemiological_Influenza_Surveillance_Standards_2014.pdf).
5. Malik MR, Mafi AR, Mahjour J, et al. Novel coronavirus infection in the Eastern Mediterranean Region: time to act. East Mediterr Health J, 2013. 19 Suppl 1:S31-8.

6. Shindo N, Briand S. Influenza at the beginning of the 21st century. Bull World Health Organ, 2012. 90(4):247-247a.

7. Polansky LS, Outin-Blenman S, Moen AC. Improved global capacity for influenza surveillance. Emerg Infect Dis, 2016. 22(6):993-1001.

8. Ortiz JR, Sotomayor V, Osvaldo C, et al. Strategy to enhance influenza surveillance worldwide. Emerg Infect Dis, 2009. 15(8): 1271-8. 